\title{
DROGENKONSUM IM LEBEN VON STUDENTEN AUF GRUND DER GEFORSCHTEN POPULATION
}

\author{
WIOLETTA KNAPIK
}

The Hugo Kołłątaj Agricultural University, Kraków, Poland

\section{ABSTRACT}

The paper „Narcotics in the Life of Students on the Basis of Investigated Population" describes the problems, which are connected with dependencies as one of greatest social problems. From ancient history to now dependencies have been accompanied by people's life. The man thanks to stimulants has been found an oblivion, the relief, the euphoria, met with other world, meditated, treated the pain and felt the consolation. It can become dependent on all things. But some from these dependencies, except the health and psychical consequences, they cause the social results too. It is happen in case of the drug addiction and other intoxicants. The life of the contemporary society, which is dominated by individualistic attitudes and subordinate to individual people's aims, there is favourable to choosing of goals, which are not compatible with the social interests. The fast pace of life, placing himself vaulting aims, there are favourable to reach for intoxicants. It was represented the druggy problem in the life of young people on the basis of inquiry research, which has been carried out among students of high schools in Cracow. The goal of this research was the presentation of attitudes of the youths today to possession and takings drugs. A main conclusion of research is that narcotics determine youths' life and they are generally accessible and accepted. The drugs don't cause the ethical problems too.

\section{UDC \& KEYWORDS}

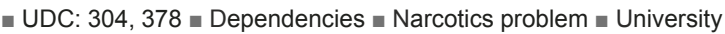
students

\section{VORWORT}

Der Begriff der Abhängigkeit wird im Folgenden verwendet im Zusammenhang mit Tätigkeiten. Tägliche Rituale bilden den wesentlichen Inhalt unseres alltäglichen Daseins. Dabei bilden Rauschmittel für die einen, die sichere Art eines Sprungbretts aus der Eintönigkeit des täglichen Lebens zu fliehen, für andere bedeutet die Abhängigkeit von ihnen die psychische und physische Basis des Lebens. Wie viele von uns ergeben sich mit der größten Hingabe den Augenblicken der täglichen Entspannung bei einer Tasse Kaffee oder einer Zigarette? Wie viele von uns kommen spätabends nach Hause nach einem anstrengenden Tag, nehmen in einem Lehnstuhl mit einem Gläschen guten Alkohol Platz? Wie viele von uns können sich das Leben ohne die tägliche Lektüre der Zeitung, das morgendliche Jogging, einen beliebten Film oder das Fernsehen nicht vorstellen? Man kann darüber streiten, wie viele unserer täglichen Rituale Gewohnheiten darstellen und wie stark wir von diesen Dingen abhängig sind. Dieses Problem illustriert das Beispiel von Tom Mc Gill, der in seinem Buch „Zu überwinden der Abhängigkeit", der seine Erfahrungen mit Abhängigsein beschreibt, die er bei seinem täglichen Jogging erfuhr. Über 10 Jahre hat der Autor fünfmal in der Woche die Strecke von fünf Meilen zurückgelegt. Darüber führte er genau Buch: die gestoppte Zeit wurde analysierte, die Anzahl der verbrauchten Kalorien und der Einfluss anderer Faktoren auf das jeweilige Ergebnisse an den einzelnen Tagen, Wochen und Jahren wurden aufgezeichnet. Man kann sich die Frage stellen - welche Faktoren wirksam wurden und inwieweit sie auf das Gefühl der Genugtuung beim Erreichen der eigenen Leistung Einfluss nahmen und wie es dazu kommt, dass die Befriedigung mit der Zeit zu einem psychischen und physischen Zwang ausartete? Der Autor denkt darüber nach, dass er das wahre Glücksgefühl gegen Ende des jeweiligen Laufes nicht öfter als ein Dutzend Mal während der Dauer dieser 10 Jahre beim Jogging verspürt hat. Dieses beweiskräftige Ereignis zwang den Autor sich einzugestehen, dass er von der Lauferei abhängig war. Als er sich nämlich eines Tages den Knöchel verstaucht hatte, war es ihm unmöglich über einen Zeitraum von 4 Wochen sein Lauftraining wieder aufzunehmen. In dieser Zeit ist er in eine Depression gefallen; er fürchtete sich, zum Fenster hinauszusehen, um keine Läufer sehen zu müssen. Dadurch ist er sich darüber klargeworden, dass er vom Jogging abhängig ist (Mc Gill 21-23). Das zeigt, dass wir vom Knabbern, vom Fernsehen, Surfen durch das Internet, vom übermäßigen Genuss von Alkohol, vom Rauchen oder Einnehmen betäubender Mittel abhängig sein können. Selbstverständlich besteht ein Grundunterschied zwischen dem Abhängigsein z.B. vom Jogging und der Abhängigkeit von Drogen. Versuchen wir aus ungezählten Beispielen des Abhängigseins gemeinsame Elemente zusammen zu stellen und zu vergleichen. Ich meine, die Gemeinsamkeit aller Verhalten, die zur Abhängigkeit führen, liegt in der Zufriedenheit und Genugtuung, welche uns diese Tätigkeit liefert. Sie kann auch in der Erwartung dieser Befriedigung durch das Ausüben der jeweiligen Tätigkeit liegen. Eben diese Erwartung der mit der Tätigkeit positiv verbundenen Genugtuung füllt uns die Zeit bis zur Entspannung aus. Diese Augenblicke der Entspannung sind oft Entschädigung, Flucht, Hingabe an Tätigkeiten, die wir im Laufe des Tages ausführen, während wir auf die Befriedigung warten. Ich bin davon überzeugt, dass die Bedeutung dieser Tätigkeiten (durch die wir befriedigt werden) gerade darin besteht, ein Gegengewicht zu den übrigen Teilen unseres Lebens zu bilden: Das ist die hauptsächliche Determination der Abhängigkeit.

Rauschgiftsucht als eines der größten gesellschaftlichen Probleme

Die Rauschgiftsucht ist eines der größten gesellschaftlichen Probleme, deren Ursache in der Entwicklung der Zivilisation liegt. Warum nehmen Menschen überhaupt Rauschmittel? Eine allgemeine Erscheinung ist die Tatsache, dass sich die Altersgrenze beständig senkt, wann nehmen wir zum ersten Mal Suchtmittel? Antworten auf diese Fragen sind nicht eindeutig.

In der Natur des Menschen schlummert die Neugier und die Sehnsucht, Neues zu entdecken. Von Kindheit an zog uns immer dasjenige am meisten an, was verboten war. Die Bibel liefert uns die Bestätigungen dafür, dass in der menschlichen Natur der Eigensinn dominiert und die Lust nach der Eroberung dessen, was verboten ist. Adam und Ewa fehlte nichts zu ihrem Glück. Sie haben dagegen aus 
Trotz und durch die übermächtige Neugier nach dem, was verboten war, alles verloren. Es scheint, dass nicht nur Menschen nach dem Entdecken unbekannter Sachen streben. Philips Robson weist auf aussagekräftige Äquivalenzen aus dem Tierreich hin: „....manche Tiere verzehren in ihrer natürlichen Umwelt manchmal Gifte und scheinen sogar daran interessiert zu sein, dieses Verhalten zu wiederholen. Man beobachtete zum Beispiel, dass Hunde an faulenden Pflanzen riechen, bis sie selbst die Koordination der Bewegungen verlieren, und dass Katzen auf ihre Fleischmahlzeit verzichteten zugunsten von Marihuana, das die unvorsichtige Eigentümerin in ihrer Reichweite ließ" (Robson 18).

Außer der Neugier fühlen Menschen immer die Notwendigkeit Sachverhalte erneut zu prüfen und Unbekanntes zu erforschen. Wir erinnern uns an Erfahrungen aus der Kindheit, als Kinder drehten wir uns im Kreise bis zur Ohnmacht. Sich einer Trance überlassen, einem wachen Traum, einem extremen Schreck beim Verhalten, all das kann sich der Menschen in der Gesellschaft leisten und durch Abhängigkeit in der KulturUmwelt tragen sie zu einem dauerhaften Element des Lebens bei.

„Bereits aus den ersten historischen Aufzeichnungen, aus der fernen Vergangenheit des menschlichen Rhodiums folgt bei nahezu allen Völkern, dass sie, sehr wahrscheinlich, schon in der Prähistorie Rauschmittel aus Pflanzen einnahmen, wodurch sie abweichende Wahrnehmungen der tatsächlichen Welt hatten, und auch zum halluzinierten Lösen der Durstes einnahmen, und dadurch den Glauben an eine Welt übernatürlicher Kräfte festigten" (Petrović 6).

Einige Wissenschaftler beschäftigten sich mit dem Problem der Rauschgiftsucht bezüglich der Fragestellung, in wieweit die Einnahme von Drogen in früher Jugend Persönlichkeitsmerkmale beeinflusst, die bewirken, dass Jugendliche zukünftig eine größere Disposition zur Abhängigkeit von Suchtmitteln zeigen können. Einfluss nehmen kann z.B. die Vorliebe für den Genuss von Alkohol, Rauchen, frühe sexuelle Erfahrungen oder eine ungeregelte Lebensweise. In der Praxis zeigt es sich dagegen, dass der Zusammenhang zwischen diesen Bereichen, die einen Hang zur Abhängigkeit suggerieren, und der Motivation zur Abhängigkeit sehr schwach ist.

Robson beruft sich auf den einzigen, formalisierten Index, der von Zuckerman erstellt wurde, der eine Skala der Wahrscheinlichkeit für die Einnahme von Drogen beschreibt. Dieser Index ergab sich auf der Grundlage von Antworten auf einen 40 Fragen umfassenden Fragebogen. Die Untersuchung, bezeichnet als „Skala der Erkundung von Empfindungen", ist durch vier Komponenten zusammengesetzt:

1. Die Lust am Erleben des Grauens und der Abenteuer,

2. die Lust extremen Durst zu überleben,

3. „der Mangel an einer Bremsen”,

4. die Flucht vor Langeweile.

Nach der Meinung von Robson ist diese Skala wenig aussagekräftig. Die Angabe eines Niveaus der Intensität der erwähnten Komponenten muss nicht schlüssig beweisen, dass die Befriedigung bestimmter Begierden einen ausreichenden Grund schafft, Suchtstoffe zu sich zu nehmen, und ausschließt, dass der Grund nicht in irgendeiner anderen Form der Befriedigung extremer Lust besteht. Dennoch hat es einen Wert die Aufmerksamkeit auf einen anderen Aspekt dieser Untersuchungen zu lenken.
Die Frage, die sich eröffnet, besteht darin, ob sich durch die Untersuchungen Gründe für das Einnehmen von Drogen durch die Jugendlichen gezeigt haben. Unter diesen Gründen haben sich die Neugier und die Lust an Spielen befunden, aber auch der soziale Druck der übrigen Mitglieder der Gruppe bildeten einen unmittelbaren Auslöser für ein Drittel der Jugendlichen und nur ein Fünftel gab an, Suchtmittel mit dem Ziel der Besserung der Stimmung eingenommen zu haben. Diese Ergebnisse haben die Voraussetzung der Untersuchung entkräftet, die davon ausging, durch die „Reduktion von Spannungen” Abhilfe zu schaffen, und die Überzeugung vertrat, dass die Mehrheit der Jugendlichen Suchtmittel mit dem Ziel einnehmen, sich von Spannungszuständen oder Depressionen zu lösen. Der Autor weist mit seinen Untersuchungen auch auf einen Zusammenhang zwischen der Einnahme von Drogen in der frühen Jugend und ihrer ständigen Einnahmen im späteren Alter hin. Robson zieht seine Schlussfolgerungen auf Grund von Tests, die in verschiedenen Universitätsstädten in der ganzen Welt über einen Zeitraum von mehreren Jahren durchgeführt wurden (Minnesota Multipfasie Inventory). Er führt die Darstellungen gleichfalls auf gesellschaftliche Faktoren zurück, die eine ungeheure Rolle im Prozess dauerhafter Drogenabhängigkeit spielen. Eine wesentliche Rolle spielt die Familie. Sind Eltern zu nachsichtig mit ihren Kindern, dann wenden sich junge Menschen, bestimmt durch das Verhalten der Familie, von klaren Verfahrensregeln ab. Eine traditionelle, konservative Erziehung schafft Gehorsam gegenüber Autoritäten und verringert die Neigung der Kinder, sich mit Verbotenem abzugeben. Der Prozess der Sozialisierung beginnt in der Familie. Sehr früh und schnell lernen Kinder gesellschaftliches Verhalten durch die Nachahmung inrer Eltern. Dort, wo in Familien ein Alkoholproblem, eine Pharmakomanie, Rauschgiftsucht oder irgendeine andere Form von Abhängigkeit besteht, gelangen Kinder schnell zur Überzeugung, dass Drogen eine Lösung für jedes Problem bilden.

„Kinder lernen vieles häufig durch Beispiele und Beobachten, dass die Mama zur Schokolade greift, wenn sie sich aufregt, und der Papa den Drink nach dem anstrengenden Arbeitstag braucht. Sie bemerken auch, dass keine Familienfeier ohne Alkohol stattfindet. Wir leben in einer Gesellschaft, die psychisch aktivierende Stoffe nutzt. Pillen unterdrücken Schwangerschaften, Arzneimittel helfen bei der Abmagerungskur, Arzneimittel bessern die eigene Stimmung auf. Für ein gutes Spiel während der ganzen Nacht hilft ecstasy oder speed" (Juczyński 29).

Das Einnehmen von Genussmittel erscheint dadurch als etwas ganz normales und ist eingeschrieben in den Zeitplan des Tages. Für noch heranwachsende Kinder in pathologischen Familien bildet das zwangsläufig den Zustand der natürlichen Umwelt, worin sie die Normen des gemeinschaftlichen Lebens lernen. Dabei wird das Verantwortungsgefühl hinsichtlich der eigenen Gesundheit zerstört und sie gewöhnen sich an die damit verbundenen finanziellen Probleme. Das Eintreten der heranwachsenden Kinder in die Welt verursacht dadurch eine Konfliktsituation zwischen ihrem bisherigen Lebensstil (in der Familie) und der Umwelt. Es eröffnet sich die Frage, inwieweit ein Kind in der Lage sein wird, seine Ansichten zu ändern, die durch seine Familie über elf oder neunzehn Jahre geprägt wurden? Es muss bemerken werden, dass ein Kind durch eine solche Erziehung jedes Verantwortungsgefühls dem eigenen Leben gegenüber beraubt wird. Führen die Eltern eine ungesunde Lebensweise, so kann sich bei einem Kind das Interesse für das Bewahren der eigenen, physischen und psychischen Gesundheit nicht festigen. Wie weit die 
Schule durch verpflichtende Lernprogramme in der Lage ist, ein wirksames Gegengewicht zu dem durch die Elternhäuser geprägten Verhalten und bestehenden Familienmuster im Verhalten der Kinder zu bilden, bleibt eine offene Frage.

Erziehung ist ein mehrphasiger und vielschichtiger Prozess. Der Glaube daran, dass Kinder sich gemäß unserer Absichten gut entwickeln, ist nicht immer begründet. Eltern rechtfertigen den notorischen Zeitmangel für Kinder gewöhnlich mit der hohen Belastung durch den Berufsalltag. Sie leben in dem Glauben, dass die Kinder ihr Engagement wahrnehmen und ihre Mühe entsprechend schätzen werden. Geld soll für Kinder einen Ausgleich für den Zeitmangel schaffen.

„Aus empirischen Untersuchungen folgt, dass für viele Personen Rauschmittel eine Arznei gegen Langeweile und Hoffnungslosigkeit sind, gegen das ewige graue Einerlei, gegen die Unbeholfenheit beim Anknüpfen von sozialen Kontakten mit Altersgenossen, die Unfähigkeit einen Freund oder einen Partner zu finden, sie sollen Arznei gegen Schwierigkeiten in der Schule und zu Hause sein. Sie ermöglichen Angst, Spannungen oder Frustration zu unterdrücken. Suchtmittel machen Vergnügen, geben Zufriedenheit, das Gefühl von Kraft, die Traumwelt zu retten und lassen die schwierige Wirklichkeit vergessen" (Muszyńska 42).

Ein weiterer Faktor, der neben der Erziehung, den von den Elternhäusern geprägten Mustern, junge Menschen zu einen Interesse an Rauschmitteln verleiten, ist die Gruppe der Altersgenossen. Jeder von uns ist Mitglied von wenigstens zwei gesellschaftlichen Gruppen: primär gebildet werden sie durch die Familie und sekundär durch die Gruppe der Altersgenossen. Das Leben in den Gruppen wird beherrscht von bestimmten Prinzipien und fordert von uns ein konformistisches (entsprechendes) Verhalten. Die Anpassung an die Normen des Gruppenlebens ist die Folge unserer Wahlen, die durch den Wunsch nach Verwirklichung unserer Hoffnungen diktiert sind. In der Gruppe fühlen wir uns besser, wir wollen angenommen werden, wir fürchten Zurückweisungen. Falls der Preis des Konformismus die Einnahme von Rauschmitteln ist, die das bestimmende Element des Gruppenlebens darstellen, werden wir diese Forderung aus Furcht vor Ausgrenzung annehmen.

„....in Übereinstimmung mit der Gültigkeit der gesellschaftlichen Theorie des Lernens ist der Einfluss der Gruppe auf den Einzelnen größer, wenn sich der Einzelne bedroht fühlt und einsam in seinem Streben. Diese Situation kann man als einen Zwangs-Konformismus bezeichnen..." (Urban 53)

Das Einnehmen von Rauschmitteln ist „Trend”; um so mehr hat es Gültigkeit zu sagen, dass die Erscheinung gefährlich ist, weil „...Drogen derart allgemein wurden, dass ihre Benutzung längst den auszeichnenden Wert verloren haben. Jeder, der Erfahrungen mit Rauschmitteln hinter sich hat, ist schon darauf aufmerksam geworden, wie eine solche Person umgeben ist vom Dunst des Geheimnisvollen, vom Widerspruchsgeist gegen traditionelle Werte oder vom romantische Gefühl eines zum Tode Verurteilten. Rauschmittel wurden Elemente der alltäglichen Wirklichkeit, sie sind allgemein zugänglich, so wie Alkohol, gebraucht im Prinzip wie ein Hilfsmittel" (Mc Gill 87).

\section{Drogenkonsum auf Grund der Forschung}

Extreme Zerstreuungen, starker Alkoholgenuss, der Drogenkonsum sind Elemente, die in einem kleinen oder größeren Grad das gemeinschaftliche Leben der gegenwärtigen Jugend bestimmen. Das Problem der www.journals.cz
Rauschgiftsucht in der gegenwärtigen Studentenschaft ist Gegenstand meiner Untersuchungen. Diese Untersuchungen habe ich im Jahr 2008 unter Studenten von Krakower Hochschulen durchgeführt. Das Ergebnis dieser Untersuchungen (unter 300 Studenten) habe ich gewonnen durch ein Verfahren, das angepasstes Schichtenverhalten abfragt. Die befragten Personen wurden hinsichtlich demographischer Merkmale differenziert :

- Nach dem Geschlecht,

- nach dem ständigen Wohnort,

- nach dem Alter (Studenten im 1., 2., 3., 4. und 5. Studienjahr).

Quantitative Untersuchungen habe ich unter Verwendung einer Rundfrage durchgeführt. Das Untersuchungsergebnis des Fragebogens wurde durch Ergebnisse aus Interviews ergänzt, die unter der Studentenschaft durchgeführt wurden, wobei sich unter den Interviewten sowohl Teilnehmer der Umfrage als auch Nichtteilnehmer befanden. Zusammengenommen haben mir die Angaben der Fragebogenaktion und der durchgeführten Interviews vielfältige Auskünfte über die Skala der Probleme, des gesellschaftlichen Verhaltens und über Veränderungen der Lebensform bezüglich des Problems der Rauschgiftsucht innerhalb der Studentenschaft geliefert. Ziel der Untersuchungen war die Darstellung des Problems der Abhängigkeit von Drogen unter Studenten. Besonders hatte ich mir vorgenommen, die Skala der Probleme zu erforschen, die sich daraus ergeben, wenn Studierende zu gemeinschaftlichen Konnotationen gedrängt werden. Ich habe die wesentliche Voraussetzung formuliert:

Rauschgifte sind Elemente im Leben der gegenwärtigen Jugend unter vier bedeutungsvollen Voraussetzungen:

1. Die traditionelle und konsequentere Erziehung in ländlichen Familien schafft eine größere Widerstandsfähigkeit gegen Bedingungen der Massenkultur.

2. Das Niveau des Wissens von den Arten und den Preisen der Drogen kann einen Zusammenhang mit dem Problem der Abhängigkeit der Jugend von diesen Rauschmitteln bilden.

3. Der unmittelbare Kontakt der befragten Personen mit Studierenden, die Drogen nehmen, hat Einfluss auf die Abhängigkeit der einzelnen Studenten und Studentinnen.

4. Die größere Duldung im Verhältnis zur Rauschgiftsucht ist ein Zeichen eines kulturellen Wandels innerhalb der gegenwärtigen Gesellschaft.

Das Thema des Artikels betrifft die Hauptthese und die ersten beiden damit verbundenen Voraussetzungen.

Zunächst das Ergebnis der Untersuchung nach dem ersten demographischen Merkmal: das Geschlecht der befragten Personen.

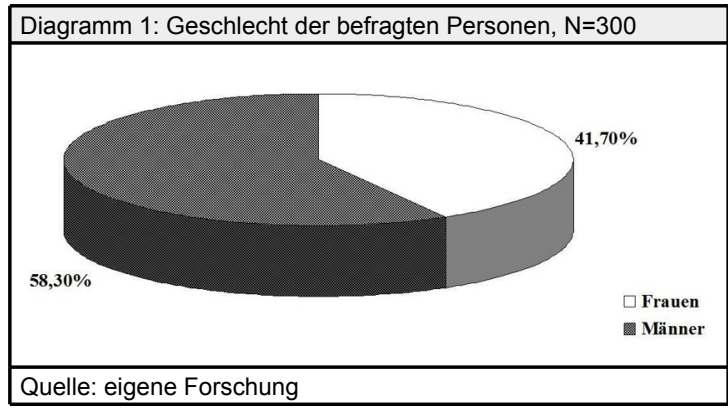




\section{DROGENKONSUM IM LEBEN VON STUDENTEN AUF GRUND DER GEFORSCHTEN POPULATION}

Notiz - In der Umfrage unter 300 Personen lag das Übergewicht bei den Männern; es sind 16,6\% (50 Personen) mehr als Frauen.

Dagegen zeigt das Ergebnis der unternommenen Umfrage bezüglich des ständigen Wohnortes der befragten Personen Folgendes.

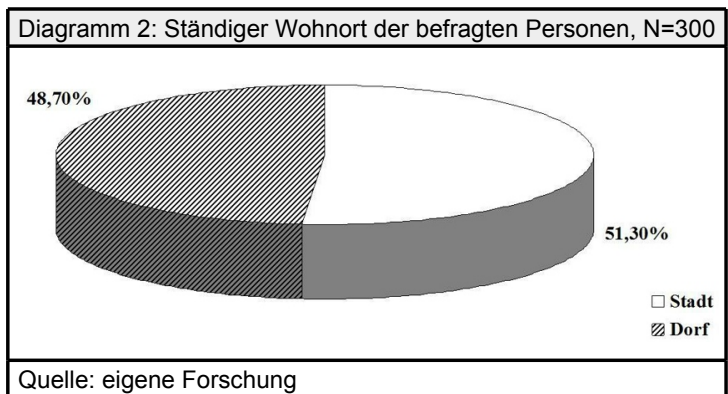

Notiz - In meinen Untersuchungen zum Problem der Abhängigkeit von Rauschmitteln unter Studentenschaft habe ich mich auf die Analyse dieser Erscheinung in Bezug auf gesellschaftliche Veränderungen konzentriert. Die Frage nach dem Preis der Drogen bezweckte, das Niveau der Kenntnis unter Jugendlichen zu erforschen. Das Wissen in Bezug des Kaufpreises einzelner Rauschmittel sagt noch nichts darüber aus, ob der Befragte diese Droge auch kaufen muss. Es muss jedoch bemerken werden, dass Personen, die sich nicht für den Kauf von Drogen interessieren, die Preise nicht kennen (so wie ein Nichtraucher die Preise von Zigaretten nicht weiß). Wichtig ist die Tatsache, dass die Jugendlichen zum überwiegenden Teil nicht berufstätig sind und für den Lebensunterhalt von den Eltern Geld bekommen, das sie gleichfalls für den Kauf der Drogen benötigen. Wie ich vermutet habe, geben die Eltern den Kindern verhältnismäßig viel Geld, um das eigene Gewissen zu beruhigen. Eine Frage nach der Art und Weise, wie das Bargeldes von den Kindern ausgegeben wird, bleibt offen.

\begin{tabular}{|l|l|l|}
\hline \multicolumn{3}{|l|}{$\begin{array}{l}\text { Tabelle 1: Antworten der befragten Personen in Bezug auf die } \\
\text { Preise der Drogen, N=300 }\end{array}$} \\
\hline Nr. & Antworten & Anzahl der Antworten \\
\hline 1 & weiß nicht & 197 \\
\hline 2 & zu hoch & 12 \\
\hline 3 & immer niedriger & 9 \\
\hline 4 & differenzierte Preise & 8 \\
\hline 5 & 30 Zloty & 14 \\
\hline 6 & 20 - 25 Zloty für ein Gramm & 7 \\
\hline 7 & 25 Zloty (Marihuana) & 6 \\
\hline 8 & 20 Zloty (Marihuana) & 5 \\
\hline 9 & 25 Zloty & 4 \\
\hline 10 & $15-20$ Zloty & 3 \\
\hline 11 & $25-50$ Zloty & 9 \\
\hline 12 & $20-400$ Zloty & 10 \\
\hline 13 & $10-200,150,200$ Zloty & 5 \\
\hline 14 & $10-500$ Zloty für ein Gramm & 7 \\
\hline 15 & ab 3 Zloty (Pillen) & 3 \\
\hline 16 & $3-50$ Zloty (Pillen) & 1 \\
\hline insgesamt & 300 \\
\hline Quelle: eigene Forschung & \\
\hline
\end{tabular}

Notiz - Wie man den Angaben entnehmen kann, kennen 1/3 der befragten Personen (103 Personen) den Preis der Drogen. Die damit verbundenen Antworten: dass die Preise
„Zu hoch”, „immer niedriger”, „differenziert“ seien (im Ganzen 29 Personen), belegt, dass die befragten Personen den aktuellen Preis der Rauschmittel kennen, aber nicht notwendiger Weise auch die Droge genommen haben müssen. Die Konstruktion des Fragebogens (mit einbezogener, offener Frage) ermöglichte es den Befragten vollständige Auskünfte zu erteilen. Die Antworten auf die Lagen von 5 bis 11 betreffen den Preise von einem Gramm Marihuana (von 15-30 Zloty), und geben Anlass zu der Vermutung, dass in der unternommenen Feldstudie wahrscheinlich diese Droge sich des "größten Erfolgs erfreut" (zusammen 48 Antworten). In den Lagen von 12 bis 14 befinden sich die teureren Drogen und die Preisobergrenze bezieht sich auf den Preis von Kokain (zusammen 22 Antworten). Die beiden letzten Lagen (1516), die den Preisen von Täfelchen betreffen, haben 4 Personen erteilt (Tabelle 1).

In der anfänglichen Phase meiner Untersuchungen bin ich von der Voraussetzung ausgegangen, dass das Niveau des Wissens von den Arten und den Preisen der Rauschmittel bezug nimmt auf das Problem der Abhängigkeit Jugendlicher von diesen Genussmitteln. Nehmen wir von dem klaren Prinzip aus: wenig oder nichts wissen wir von einer Sache, die nicht Gegenstand unseres Interesses oder Begehrens ist. Etwa ein Drittel der Befragten kennen die Preise der Drogen. Daraus kann man sicher schließen, dass dieser Teil der Jugend überhaupt an Drogenkonsum interessiert ist.

Etwa ein Drittel der Befragten (30,3\%) wissen nicht, welche Droge stärker wirkt. Der übrige Teil der befragten Personen $(69,7 \%)$ gab Antworten, die bereits unter dem Blickwinkel der Zahlwerte in der Tabelle 2 vorgestellt wurden. Die Untersuchungsfrage: „Was für ein Suchtmittel ist nach Deiner Meinung stärker?" war eine der offenen Fragen. Den befragten Personen wurden keine Antworten vorgegeben. Auf die Fragen nach dem Wissen bei Frauen über die stärkste Droge, hat die Mehrheit 94 Personen (31,3\% der Gesamtheit) geantwortet. Die Antworten dieser Personen stimmen mit der Wahrheit überein. Die befragten Frauen verfügen über ein Wissen von den stärksten und schädlichsten Drogen, die sowohl physisch wie psychisch abhängig machen. Kokain, so die befragten Frauen, wurde als Droge gesehen, die sowohl physisch wie psychisch abhängig macht, was, entsprechend der Zahl der Antworten, dem dritten Platz entsprach (8,7 \%). Marihuana und LSD verstanden als „weiche” Droge, ist im ganzen von 24 Befragungspersonen (8\%) als stärkste bezeichnet worden. Die interessanteste Schlussfolgerung kann man aus der Antwort von 40 Befragungspersonen (13,3\%) herausschälen, die als stärkste Droge das Amphetamin genannt haben. Das Amphetamin wird de facto nicht zu den harten Drogen gezählt, da sie nicht physisch abhängig macht und nicht unmittelbar Folgen hat wie der Platz in dem Zufall Heroin oder Kokain. Diese Droge ist dagegen sehr beliebt unter Schülern und Studenten, da sie als Mittel den Organismus bei ermüdender Geistesarbeit unterstützt und mobilisiert. Diese Popularität ist vielleicht der Grund, dass sich das Amphetamin auf Platz zwei der Liste der stärksten Rauschmittel befindet. Das soll die Aufmerksamkeit darauf lenken, dass Trends der Massenkultur in der gegenwärtigen Welt hauptsächlich von den Vereinigten Staaten von Amerika bestimmen werden. So steht zu erwarten und zu befürchten, dass eine große Welle des Problems, die Verbreitung von Drogen, noch vor uns liegt. Ich habe im Sinne des Allgemeinwissens, der Zugänglichkeit und der Ungleichbehandlung der Drogen bereits weltanschauliche Änderungen in unserem Land, das Problem der Rauschgiftsucht betreffend, bemerkt (Tabelle2). 


\section{DROGENKONSUM IM LEBEN VON STUDENTEN AUF GRUND DER GEFORSCHTEN POPULATION}

\begin{tabular}{|l|l|}
\hline \multicolumn{2}{|l|}{$\begin{array}{l}\text { Tabelle 2: Welche Droge ist nach Meinung der befragten Person } \\
\text { stärker?, N=300 }\end{array}$} \\
\hline Antworten & Anzahl der Antworten \\
\hline Heroin & 94 \\
\hline Amphetamin & 40 \\
\hline Kokain & 26 \\
\hline Marihuana & 14 \\
\hline LSD & 10 \\
\hline jede Droge ist stark & 9 \\
\hline Metaamphetamin & 6 \\
\hline Extasy & 3 \\
\hline Brown Sugar & 2 \\
\hline andere & 5 \\
\hline weiß nicht & 91 \\
\hline insgesamt & 300 \\
\hline Quelle: eigene Forschung & \\
\hline
\end{tabular}

Notiz - Die Tatsache, dass jeder dritte befragte Student Drogen kennt und einen Preis zu nennen vermag, deutet auf den Markt der Rauschmittel hin. Einige von den Befragten gaben einen Preis für $1 \mathrm{Gramm}$ einer Droge an, andere tauschten die Preisangaben aus oder nannten auch einen angenäherten Preis konkreter Drogen. Die Mehrheit der befragten Personen (69,7\%) vermochte Antworten auf die Fragen nach der Stärke von Rauschgiften zu geben. Daraus ziehe ich den Schluss, dass die von mir aufgestellt Voraussetzung, dass das Niveau des Wissens von den Arten und den Preisen von Drogen mit dem Problem der Abhängigkeit der Jugend von diesen Rauschmitteln einen Zusammenhang bildet, bestätigt werden kann. Im Durchschnitt scheinen die Studenten die Namen der Drogen zu kennen, die am häufigsten genannt werden. In meinen Untersuchungen haben 94 Personen aus der Gruppe von 300 Befragten die richtigen Bezeichnungen für die stärksten Drogen angegeben. Diejenigen Personen, die an Drogen nicht interessiert sind, hätten nach meiner Überzeugung, Probleme bei der Angabe von Drogenpreisen gehabt. Die Untersuchungen haben aber deutlich gemacht, dass ein Drittel der befragten Personen den Preis der Drogen kennen.

Eine abwechselnde Voraussetzung ist: Die traditionelle und konsequentere Erziehung in ländlichen Familien schafft eine größere Widerstandsfähigkeit gegen die Forderungen der Massenkultur. Die gegenwärtige Massenkultur ist gekennzeichnet durch: den Kult des Geldes, den Jugendkult, ein Leben im Zustand extremer Empfindungen, sie werden unterstützt von Alkohol, verschiedenen Genussmittel, Chemotherapeutika (synthetische Mittel, unter denen sich auch Drogen befinden). Ich meine, dass die Erziehung den entscheidenden Einfluss darauf hat, in welcher Weise die Jugendlichen ihr Leben steuern und inwieweit sie dem Druck der Umgebung erliegen. Die folgende Übersicht belegt das Niveau des Interesses der Studentenschaft an Drogen. Das Ziel der Untersuchung war es, den Einfluss der Erziehung auf das Verhalten der befragten Personen sichtbar zu machen. Die einzige Kategorie dieser Antwort habe ich für die Untersuchung in der Frage nach dem dauerhaften Wohnort der Jugend in der Stadt oder auf dem Lande aufgeteilt.

Die genannten Kategorien waren eingerückt in der Zusammenstellung von Fragen und wurden von mir erst während der Analysen der Antwort von der offenen Frage abgesondert: Was für Schritte würden die befragten Personen unternehmen, um an Drogen zu gelangen? Zwei Kategorien der Antwort, nämlich: „keine” und „weiß nicht”, weisen synonym auf das Desinteresse der Befragten an den Drogen hin. Im Ergebnis überwogen beide Varianten bei

den Antworten der befragten Personen, die vom Lande stammten. Entsprechend verschoben sich die Ergebnisse von $3,9 \%$ und $8,9 \%$ der Antworten im Verhältnis zu der befragten Personen, die aus der Stadt stammen. Auf Grund Datenauswertungen neige ich zu der Annahme, dass die Kategorien "das Telefon" und "Dealer” identisch sein können. Herausgefunden werden sollte, wie die Drogen zu erwerben sind. Dann würde das Telefon die Bedeutung haben, sich mit der Person zu verbinden, die ihnen den Stoff liefern könnte oder helfen könnte, einen Lieferanten von Drogen zu bekommen. In gewissem Sinne kann man diese Personen "dealer" nennen. Beide dieser Kategorien haben 22,8\% der Antworten der befragten Personen aus der Stadt ausgemacht und nur 10,9\% der Antworten von Befragten vom Lande. Fast um die Hälfte kleiner ist die Gruppe der befragten Personen vom Lande, die diese bequemste Möglichkeit des Erwerbens der Drogen nutzen würde. In der Kategorie „ich frage Bekannte, Kollegen” habe ich auch deutlich weniger Antworten seitens der (etwa 5,2\% weniger im Verhältnis zu der städtischen) Jugendlichen vom Lande benommen (Tabelle 3).

\begin{tabular}{|c|c|c|c|c|c|c|}
\hline $\begin{array}{l}\text { Tabelle 3: Was } \\
\text { unternehmen, un }\end{array}$ & $\begin{array}{l}\text { ür Schritte } \\
\text { an Drogen z }\end{array}$ & $\begin{array}{l}\text { würc } \\
\text { u ge }\end{array}$ & $\begin{array}{l}\text { die } \\
\text { gen? }\end{array}$ & befr & $n \mathrm{P}$ & sonen \\
\hline Antworten & Insgesamt & $\%$ & Stadt & $\%$ & Dorf & $\%$ \\
\hline Keine & 105 & 35 & 51 & 33,1 & 54 & 37 \\
\hline $\begin{array}{l}\text { Ich frage } \\
\text { Bekannte, } \\
\text { Kollegen }\end{array}$ & 47 & 15,7 & 28 & 18,2 & 19 & 13 \\
\hline Weiß nicht & 46 & 15,3 & 17 & 11 & 29 & 19,9 \\
\hline $\begin{array}{l}\text { es fehlt eine } \\
\text { Entwort }\end{array}$ & 40 & 13,3 & 20 & 13 & 20 & 13,7 \\
\hline Das Telefon & 33 & 11 & 23 & 15 & 10 & 6,8 \\
\hline Dealer & 18 & 6 & 12 & 7,8 & 6 & 4,1 \\
\hline Internet & 4 & 1,3 & 1 & 0,6 & 3 & 2 \\
\hline $\begin{array}{l}\text { In der } \\
\text { Wohnsiedlung }\end{array}$ & 2 & 0,7 & 0 & 0 & 2 & 1,4 \\
\hline $\begin{array}{l}\text { Es sind } \\
\text { vorhanden }\end{array}$ & 3 & 1 & 2 & 1,3 & 1 & 0,7 \\
\hline $\begin{array}{l}\text { Disco,Club,Pub, } \\
\text { Fitnessstudio }\end{array}$ & 2 & 0,7 & 0 & 0 & 2 & 1,4 \\
\hline Insgesamt & 300 & 100 & 154 & $100 \%$ & 146 & $100 \%$ \\
\hline $\begin{array}{l}N=154(100 \%-i \\
N=146(100 \%-i\end{array}$ & $\begin{array}{l}\text { der Stadt } \\
\text { Dorf woh }\end{array}$ & hne & $\begin{array}{l}\text { len } \mathrm{Be} \\
\text { Befras }\end{array}$ & $\begin{array}{l}\text { agten) } \\
\text { en) }\end{array}$ & & \\
\hline Quelle: eigene F & rschung & & & & & \\
\hline
\end{tabular}

Notiz - Der Fragebogen wurden auf eine Art konstruiert, um die Befragten nicht unmittelbar nach ihren Erfahrungen mit Drogen zu fragen. Ich weiß aus Erfahrung, dass zu viele vertrauliche und persönliche Fragen die befragten Personen in die Verlegenheit führen und schließlich nur ausweichende Antworten verursachen. Die Befragten wurden nach ihrer Meinung über nahe Bekannten, Kollegen und Kameradinnen gebeten. Nach meiner Ansicht wird innerhalb des Bekanntenkreises ehrlicher über die Einschätzung und die eigene Haltung angesichts von Problemen beim Drogenkonsums gesprochen.

Um auf den Unterschied im Zusammenhang des Denkens der ländlichen und städtischen Jugend zurückzukommen, stelle ich im Folgenden die Befunde vor, die auf die Erfahrungen der befragten Personen hinsichtlich der Einnahme von Drogen im Kreis ihrer Bekannten Bezug nehmen (Tabelle 4).

Notiz. Die Angaben bestätigen die von mir aufgestellte Voraussetzung, dass die strengere Erziehung in Familien auf dem Lande das Muster des Verhaltens der Jugend beeinflusst, die kritischer ist im Verhältnis zu dem in der Stadt aufgekommenen Lebensstil. Bis zu $16,4 \%$ ist die 


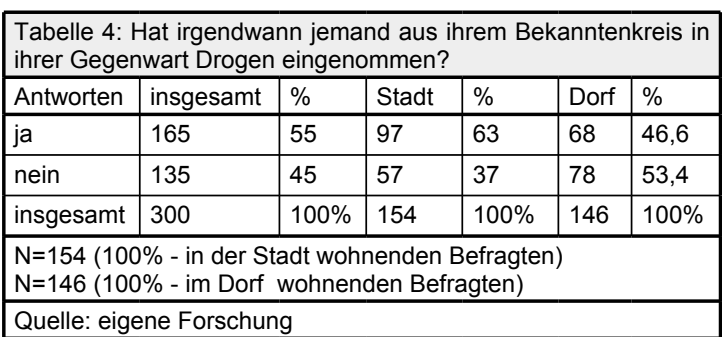

Gruppe der befragten Personen kleiner, die vom Lande kommend weniger unmittelbaren Kontakt mit Personen hatten, die Drogen einnehmen. Zu diesen Ausführung bilden Schlussfolgerungen eine Ergänzung, die sich aus der Analysen der Antworten ergeben, die die Personen nach den Preisen von Drogen befragten. Die Antwort: „ich weiß nicht" auf die Frage nach dem Preis der Rauschmittel soll auslegt werden, unter besonderer Berücksichtigung der Gefährdung der Befragten durch das Problem der Drogen. Über $20,2 \%$ lag die Zahl der vom Lande stammenden Personen über der der städtischen, befragten Personen, die diese Variante als Antwort gewählt hatten. In der Gruppe der Kategorie: „zu hoch”, „immer niedriger”, "differenziert" waren 9,5\% der Antworten weniger auf der Seite derjenigen, die dauerhaft auf dem Lande wohnten. Entsprechendes taucht auch in den übrigen Ergebnissen auf, dort, wo die befragten Personen die konkrete Preise für Drogen austauschen sollten, lagen die Antworten um 10,7\% höher als bei den befragten Jugendlichen vom Lande.

\section{Endung}

Das Problem der Rauschmittel ist ein Zivilisations - Problem, das besonders gefährlich ist im Kontext der Abhängigkeit der Kindern und der Jugendlichen. Die Besonderheit dieses Problems verlässt sich auf Ursachen der Einnahme von Drogen nur im Kontext genetischer Bedingen vermutet, aber vor allem in dem breiten gemeinschaftlichen Kontext. Die gegenwärtige Welt gestaltet das Leben anstrengend, die Menschen überschreiten voll der Empfindungen alle Möglichkeiten unserer Sinne, das veranlasst sie nach anderen Lösungen zu suchen. Das Angebot kommt nicht nur hinter der Nachfrage, sondern durch immer neue Medien produzieren wir die Illusion einer Wirklichkeit mit. Die Welt des Geschäftes schafft Nachfrage auf ständig neuen Wegen in der Auseinandersetzung des Menschen mit eigenen Schwächen, mit Stress, dem Minderwertigkeitsgefühl. Die Wirkung der Drogen auf die Jugend kann man besser verstehen, wenn alle Faktoren des gesellschaftlichen Lebens in der gegenwärtigen Welt berücksichtigen wird. In dem Bericht Päpstlichen Stuhles, über Sekten lesen wir, dass junge Menschen prüfen „....verschiedenerlei Formen der Großmächte, der Konflikte, der ökologischen Drohung, der nuklearen Vernichtung und ihrer Handhabung. Sie fühlen die Frustration, die Entwurzelung, die Beraubung der Hoffnung und der Motivation. Sie fühlen sich in einer Einöde und verloren in der Anonymität und der Isolierung, herabgestoßen an den Rand gedrängt, unverstanden, verraten, bedrückt, betrogen, nicht gehört und nicht angenommen. Sie sind enttäuscht, vor allem durch das 20. Jahrhundert - von der ewige Zivilisation der Industrialisierung, der Welt des Geschäftes, von Erziehungssystemen, von den Vorschriften und den Praktiken der Kirche und enttäuscht von der Politik der Regierungen" (Nowak 225).

Der gesellschaftliche Kontext der Rauschgiftsucht ergänzt die Pathologie des Lebens als Ergebnis vom Missbrauch der Rauschmittel. Alle erwähnten Faktoren werden unterstützt vom Einfluss der Massenkultur, die unausgesetzt die Mode „für das Konsumieren” in Schwung hält und sie verletzt am zartesten Punkt, dem Ego der Kinder und Jugendlichen.

\section{LITERATURVERZEICHNIS}

1. Juczyński, Z., (2002). Narkomania. Podręcznik dla nauczycieli, wychowawców i rodziców. Wydawnictwo Lekarskie PZWL, Warszawa, 29.

2. Mc Gill, T., (2007). Przezwyciężyć uzależnienie. Wydawnictwo Zysk i S-ka, Poznań, 21-23, 87.

3. Muszyńska, A., (2004). Narkomani sprawcy czynów karalnych. Kantor Wydawniczy Zakamycze, Kraków, 42.

4. Nowak, A.J. OFM, Hrsg., (1999). Satanizm, rock, narkomania, seks. Redakcja Wydawnictw Katolickiego Uniwersytetu Lubelskiego, Lublin, 225.

5. Petrović, S.P., (1988). Narkotyki i człowiek. Wydawnictwo Iskry, Warszawa, 6.

6. Robson, P., (1997). Narkotyki. Wydawnictwo Medycyna Praktyczna, Kraków, 18.

7. Urban, B., (1998). Zachowania dewiacyjne młodzieży. Wydawnictwo UJ, Kraków, 53. 